\title{
Uso da aromaterapia como tratamento adjuvante na redução de sintomas de indivíduos com ansiedade
}

\author{
Use of aromatherapy as an adjunct treatment to reduce symptoms in individuals with anxiety \\ Uso de aromaterapia como tratamiento complementario para reducir los síntomas en personas con
} ansiedad

\begin{abstract}
Resumo
Introdução: A aromaterapia é uma prática multiprofissional que pode atuar de forma benéfica na busca do reequilíbrio físico e também emocional das pessoas que utilizam essa terapêutica. Objetivo: Analisar a aplicação da aromaterapia como tratamento coadjuvante no tratamento de distúrbios de ansiedade. Métodos: Revisão integrativa da literatura, realizada com artigos disponíveis na BVS, SciELO, LILACS e MEDLINE, com um intervalo de anos de 2010 a 2020. Foram selecionadas as pesquisas de acordo com o nível hierárquico de evidencia científica para estudos intervencionistas. Resultados: Foram encontrados estudos que apontaram o uso da aromaterapia na redução de parâmetros como frequência cardíaca (FC); frequência respiratória (FR); pressão arterial (PA); e, pressão arterial média (PAM) em indivíduos com ansiedade; como também redução no nível de estresse e aumento no relaxamento muscular, proporcionando melhor qualidade de vida para esses indivíduos. Conclusão: Houve resultados positivos na utilização da aromaterapia como tratamento coadjuvante na redução de sintomas de ansiedade.
\end{abstract}

Palavras-chave: Aromaterapia; Ansiedade; Terapias Complementares; Enfermagem.

\begin{abstract}
Introduction: Aromatherapy is a multidisciplinary practice that can act in a beneficial way in the search for physical and emotional rebalancing of people who use this therapy. Objective: To analyze the application of aromatherapy as an adjunct treatment in the treatment of anxiety disorders. Methods: Integrative literature review, carried out with articles available in the VHL, SciELO, LILACS and MEDLINE, with a range of years from 2010 to 2020. Researches were selected according to the hierarchical level of scientific evidence for interventional studies. Results: Studies were found that showed the use of aromatherapy to reduce parameters such as heart rate (HR); respiratory rate (RR); blood pressure (BP); e, mean arterial pressure (MAP) in individuals with anxiety; as well as a reduction in the level of stress and an increase in muscle relaxation, providing a better quality of life for these individuals. Conclusion: There were positive results in the use of aromatherapy as an adjunctive treatment in reducing anxiety symptoms.
\end{abstract}

Keywords: Aromatherapy; Anxiety; Complementary Therapies; Nursing.

\section{Resumen}

Introducción: La aromaterapia es una práctica multidisciplinar que puede actuar de forma beneficiosa en la búsqueda del reequilibrio físico y emocional de las personas que utilizan esta terapia. Objetivo: Analizar la aplicación de la aromaterapia como tratamiento coadyuvante en el tratamiento de los trastornos de ansiedad. Métodos: Revisión bibliográfica integradora, realizada con artículos disponibles en la BVS, SciELO, LILACS y MEDLINE, con un 
rango de años de 2010 a 2020. Las investigaciones fueron seleccionadas de acuerdo al nivel jerárquico de evidencia científica para estudios intervencionistas. Resultados: Se encontraron estudios que mostraron el uso de aromaterapia en la reducción de parámetros como la frecuencia cardíaca (FC); frecuencia respiratoria (RR); presión arterial (PA); e, presión arterial media (PAM) en individuos con ansiedad; así como una reducción del nivel de estrés y un aumento de la relajación muscular, proporcionando una mejor calidad de vida para estos individuos. Conclusión: Se obtuvieron resultados positivos en el uso de aromaterapia como tratamiento coadyuvante en la reducción de los síntomas de ansiedad.

Palabras clave: Aromaterapia; Ansiedad; Terapias Complementarias; Enfermería.

\section{Introdução}

A ansiedade é um distúrbio que afeta cerca de 9,3\% (18.657.943) da população brasileira, estando, a nível mundial, em destaque como uma das patologias que causam mais incapacidade em vários aspectos da vida das pessoas, aumentando a carga global de doenças. As dificuldades de acesso aos serviços de saúde, tratamento, reabilitação, e as questões do estigma que cerca os portadores de ansiedade ainda se fazem presentes nos tempos de hoje e se apresenta como algo desvantajoso na vida das pessoas que possuem esse transtorno (OPAS, 2017).

Além desse fator, esse tipo de distúrbio afeta de forma significativa as relações interpessoais levando a sérias disfunções no trabalho, meio familiar e social. Estudos apontam que a ansiedade é responsável por cerca de 800 mil mortes de jovens entre 15 e 29 anos, configurando-se assim como um problema de saúde pública (OPAS, 2017; Pereira et al. 2019).

Atualmente, esse problema de saúde está entre os transtornos mais prevalentes identificados entre os pacientes que são acolhidos na atenção secundária à saúde mental. Porém, grande parte poderia ter seu tratamento e controle na atenção primária. (American Psychiatric Association, 2014).

Sendo assim, é indispensável à otimização do acolhimento integral, humanização do serviço e a atuação da equipe multidisciplinar, que possam vir identificar as particularidades e as necessidades do indivíduo em questão, para que seja escolhido o melhor tratamento para o mesmo (American Psychiatric Association, 2014; Onocko-Campos, 2019).

Salienta-se que os psicofármacos são os principais recursos terapêuticos escolhidos para o tratamento da ansiedade. Eles se encontram entre os medicamentos de uso mais prevalentes seguidos dos antidepressivos, sendo os benzodiazepínicos os medicamentos com maior número de prescrição a nível mundial. Porém, isso se configura como algo desvantajoso, visto que esses podem causar efeitos colaterais e dependência, podendo diminuir assim a qualidade de vida dos pacientes (Leonardo et al. 2017).

Uma das práticas que podem otimizar o tratamento de transtornos ansiosos é a aromaterapia, que é descrita como uma prática multiprofissional, fitoterápica e não farmacológica, que pode atuar de forma benéfica na busca do reequilíbrio físico e também emocional das pessoas que utilizam essa terapêutica (Brasil, 2018; Hoare, 2010; Malta \& Lemos, 2019).

Além disso, essa prática, não farmacológica, é considerada uma das melhores opções para a amenização de sintomas relacionados ao desequilíbrio emocional do indivíduo. Sua alta eficácia está correlacionada com fato da absorção dos Óleos Essenciais (OE) acontecerem de várias formas, seja ela por meio da via inalatória; através de uso tópico, quando entra em contato com a pele; como também através da ingestão (Brasil, 2018; Hoare, 2010; Machado \& Júnior, 2011).

Pensando nisso, esse trabalho estabeleceu a seguinte pergunta norteadora: “O que os estudos trazem acerca de resultados alcançados em indivíduos com ansiedade que utilizaram a aromaterapia como tratamento adjuvante?”. Para responder a pergunta norteadora, essa pesquisa tem como objetivo analisar a aplicação da aromaterapia como tratamento coadjuvante no tratamento de distúrbios ansiosos; quais os efeitos dessa terapêutica; além de entender qual a atuação dos enfermeiros na aplicação deste tratamento. 


\section{Metodologia}

Essa pesquisa trata-se de um estudo bibliográfico, descritivo, do tipo revisão integrativa da literatura. Esse tipo de pesquisa bibliográfica tem como objetivo selecionar e sintetizar os principais estudos acerca do tema definido inicialmente. As pesquisas selecionadas não precisaram necessariamente ter apenas uma abordagem metodológica, isso possibilitou que o agrupamento dos achados acerca do tema fossem os mais variados possíveis (Mendes et al. 2013; Sousa et al. 2010).

De tal maneira, para elaboração da pergunta norteadora foi utilizada a estratégia PICO, tal sigla é um acrônimo para as palavras: Paciente, Intervenções, Comparação e "outcomes", que significa desfecho (Santos et al. 2007). Assim no tema proposto estabeleceu-se P- Indivíduos diagnosticados com distúrbios ansiosos; I- Aromaterapia como recurso terapêutico; CPacientes que fizeram tratamento com a inserção de tal técnica; O- Resultados obtidos.

Posteriormente, foi realizada a busca pelos estudos acerca do tema no período de 2010 a 2020; no idioma português e inglês; no portal regional da Biblioteca Virtual de Saúde (BVS), Scientific Electronic Library Online (SciELO), Literatura Latino Americana e do Caribe em Ciências da Saúde (LILACS), Medical Literature Analysis and Retrieval Sistem Online (MEDLINE) e Base de Dados em Enfermagem (BDENF).

Para a busca dos estudos no idioma português foram utilizados os Descritores em Ciências da Saúde (DeCS) e no idioma inglês o Medical Suject Headings (MeSH). Foi utilizado o operador booleno AND, para a realização das possíveis combinações entre os descritores: Aromaterapia; Ansiedade; Terapias Complementares; Enfermagem.

Além disso, para seleção da amostra do estudo foram atribuídos os seguintes critérios de inclusão: estudos na íntegra e originais, que estivessem entre o período de 2010 a 2020 e que estivessem dentro da temática de estudo. Foram excluídos artigos que fugiram do tema proposto, monografias, dissertações, trabalhos apresentados em anais de congressos, cartas de opinião, teses, editoriais e resumos.

Após essa etapa, foram analisados os títulos dos artigos, sendo esses de inteira relação com o tema da presente pesquisa, contemplando responder os objetivos do estudo. Foram lidos os resumos e posteriormente os textos na íntegra, sendo excluídos da pesquisa aqueles que não estavam de acordo com o tema.

Foram selecionadas as pesquisas de acordo com o nível hierárquico de evidencia científica para estudos intervencionista, sendo representada por: Nível I - revisão sistemática ou metanálise; Nível II - estudos controlados, individual com delineamento experimental; Nível III - estudos controlados sem randomização; Nível IV - estudos caso-controle ou de coorte; Nível V - revisão sistemática de estudos qualitativos ou descritivos; Nível VI - estudos qualitativos ou descritivos e Nível VII - opiniões ou consensos (Bernardo, 2011; Galvão, 2006).

Para seleção das pesquisas, foram utilizadas as recomendações dos Principais Itens para Relatar Revisões Sistemáticas e Meta-análises (PRISMA), na qual utilizou-se métodos claros e objetivos para identificar e selecionar as pesquisas que foram relevantes (Galvão et al. 2015); como apontadas na Figura 1. 
Figura 1 - Fluxograma adaptado do modelo PRISMA.

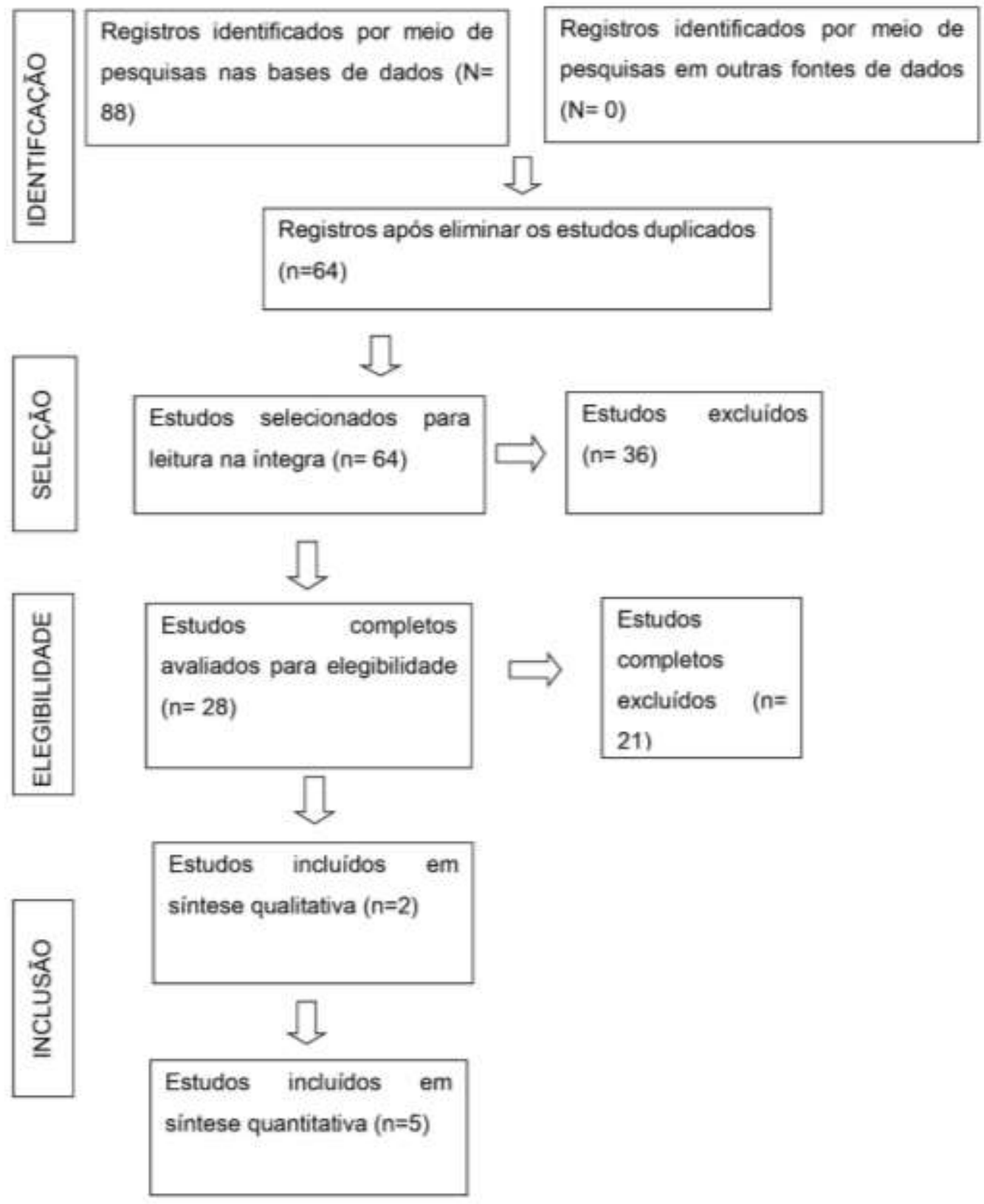

Fonte: Dados da pesquisa (2020).

\section{Resultados}

Foram selecionados sete artigos científicos para compor a amostra final da pesquisa. Tal seleção se deu a partir da busca realizada nas bases de dados delimitadas previamente, obedecendo os critérios metodológicos. Desses, um estava disponível na base de dados da SciELO, dois na MEDLINE, dois na BDENF e dois na LILACS. Os resultados foram organizados em dois quadros: o Quadro 1 traz as informações dos autores, títulos, objetivos, periódicos e ano; e o Quadro 2, a modalidade da pesquisa, nível de evidência e principais resultados encontrados nos artigos selecionados. 
Quadro 1 - Síntese dos estudos sobre a utilização da aromaterapia como método de dimiuição do nível de ansiedade.

\begin{tabular}{|c|c|c|c|c|}
\hline ID & Autores & Títulos & Objetivos & Periódico/Ano \\
\hline 01 & $\begin{array}{l}\text { Wells } \quad \text { BM; } \\
\text { Nightingale } \quad \text { LM; } \\
\text { Derby DC; Salsbury } \\
\text { SA; Lawrence D }\end{array}$ & $\begin{array}{l}\text { Aromatherapy for test anxiety in } \\
\text { chiropractic students: A feasibility } \\
\text { study }\end{array}$ & $\begin{array}{l}\text { Avaliar a viabilidade de } \\
\text { estudantes de Quiropraxia } \\
\text { estudo } \\
\text { envolvendo } \text { um } \\
\text { redução da ansiedade. }\end{array}$ & J Chiropr Educ/2020 \\
\hline 02 & $\begin{array}{l}\text { Dias SS; Domingos } \\
\text { TS; Braga EM }\end{array}$ & $\begin{array}{l}\text { Aromaterapia para a ansiedade e } \\
\text { estresse de professores de } \\
\text { enfermagem }\end{array}$ & $\begin{array}{l}\text { Investigar a efetividade do uso da } \\
\text { aromaterapia com os óleos essenciais } \\
\text { de lavanda (Lavandula angustifolia) } \\
\text { ou ylang-ylang (Cananga odorata), } \\
\text { associada à massagem, para o alívio } \\
\text { da ansiedade e do estresse. }\end{array}$ & $\begin{array}{l}\text { Rev enferm UFPE on } \\
\text { line } / 2019\end{array}$ \\
\hline 03 & $\begin{array}{l}\text { Son HK; So W; Kim } \\
\text { M }\end{array}$ & $\begin{array}{l}\text { Effects of Aromatherapy Combined } \\
\text { with Music Therapy on Anxiety, } \\
\text { Stress, and Fundamental Nursing } \\
\text { Skills in Nursing Students: A } \\
\text { Randomized Controlled Trial }\end{array}$ & $\begin{array}{l}\text { Identificar intervenções eficazes para } \\
\text { aliviar emoções negativas } \\
\text { relacionadas aos testes, melhorando } \\
\text { assim a enfermagem desempenho } \\
\text { acadêmico geral dos alunos e saúde } \\
\text { mental }\end{array}$ & $\begin{array}{l}\text { Int. J. Environ. Res. } \\
\text { Public Health/2019 }\end{array}$ \\
\hline 04 & $\begin{array}{l}\text { Domingos TS; Braga } \\
\text { EM }\end{array}$ & $\begin{array}{l}\text { Massagem com aromaterapia: } \\
\text { efetividade sobre a ansiedade de } \\
\text { usuários com transtornos de } \\
\text { personalidade em internação } \\
\text { psiquiátrica }\end{array}$ & $\begin{array}{l}\text { Investigar a efetividade da } \\
\text { aromaterapia associada à massagem } \\
\text { sobre a ansiedade de pacientes com o } \\
\text { diagnóstico de TP durante a } \\
\text { internação psiquiátrica. }\end{array}$ & $\begin{array}{l}\text { Rev Esc Enferm } \\
\text { USP/2015 }\end{array}$ \\
\hline 05 & $\begin{array}{ll}\text { Gnatta JR; } & \text { Piason } \\
\text { PP; Lopes } & \text { CLBC; } \\
\text { Rogenski } & \text { NMB; } \\
\text { Silva MJP } & \end{array}$ & $\begin{array}{l}\text { Aromaterapia com ylang ylang para } \\
\text { ansiedade e autoestima: estudo } \\
\text { piloto }\end{array}$ & $\begin{array}{l}\text { Verificar se o uso do óleo essencial } \\
\text { de ylang ylang por meio de aplicação } \\
\text { cutânea ou inalatória altera a } \\
\text { percepção da ansiedade e da } \\
\text { autoestima e os parâmetros } \\
\text { fisiológicos como pressão arterial e } \\
\text { temperatura. }\end{array}$ & $\begin{array}{l}\text { Rev Esc Enferm } \\
\text { USP/2014 }\end{array}$ \\
\hline 06 & $\begin{array}{l}\text { Mendes DS, Moraes } \\
\text { FS, Lima GO, Silva } \\
\text { PR, Cunha TA, } \\
\text { Crossetti MGO, et al. }\end{array}$ & $\begin{array}{l}\text { Benefícios das práticas integrativas } \\
\text { e complementares no cuidado de } \\
\text { enfermagem }\end{array}$ & $\begin{array}{l}\text { Caracterizar os benefícios das } \\
\text { práticas integrativas } \\
\text { complementares no cuidado de } \\
\text { enfermagem. }\end{array}$ & $\begin{array}{l}\text { Journal } \\
\text { NPEPS/2019 }\end{array}$ \\
\hline 07 & $\begin{array}{l}\text { Lyra CS, Nakai LS, } \\
\text { Marques AP }\end{array}$ & $\begin{array}{l}\text { Eficácia da aromaterapia na redução } \\
\text { de níveis de estresse e ansiedade em } \\
\text { alunos de graduação da área da } \\
\text { saúde: estudo preliminar }\end{array}$ & $\begin{array}{l}\text { Verificar a eficácia de aromaterapia } \\
\text { na diminuição de níveis de estresse e } \\
\text { ansiedade (traço e estado) de alunos } \\
\text { de graduação de cursos na área da } \\
\text { saúde }\end{array}$ & $\begin{array}{l}\text { Fisioterapia } \\
\text { Pesquisa/ } 2010\end{array}$ \\
\hline
\end{tabular}


Quadro 2 - Síntese dos estudos sobre a utilização da aromaterapia como método de dimiuição do nível de ansiedade.

\begin{tabular}{|c|c|c|c|c|}
\hline ID & Título & $\begin{array}{l}\text { Modalidade da } \\
\text { Pesquisa }\end{array}$ & $\begin{array}{l}\text { Nível de } \\
\text { Evidência }\end{array}$ & Síntese dos Resultados \\
\hline 01 & $\begin{array}{l}\text { Aromatherapy for test anxiety in } \\
\text { chiropractic students: A feasibility } \\
\text { study }\end{array}$ & $\begin{array}{l}\text { Ensaio clínico } \\
\text { randomizado }\end{array}$ & Nível II & $\begin{array}{l}\text { A grande maioria dos estudantes demonstraram } \\
\text { disposição em utilizar a aromaterapia no futuro como } \\
\text { técnica para reduzir os níveis de ansiedade. }\end{array}$ \\
\hline 02 & $\begin{array}{l}\text { Aromaterapia para a ansiedade e } \\
\text { estresse de professores de } \\
\text { enfermagem }\end{array}$ & $\begin{array}{l}\text { Estudo } \\
\text { quantitativo, } \\
\text { exploratório- } \\
\text { descritivo }\end{array}$ & Nível VI & $\begin{array}{l}\text { Houve redução da pressão arterial e em outros } \\
\text { parâmetros como frequência cardíaca e saturação de } \\
\text { oxigênio, porém os últimos citados não apresentaram } \\
\text { tanta relevância significativa. O grupo de fizeram as } \\
\text { sessões com o OE de ylang ylang apresentaram redução } \\
\text { nos níveis de estresse comparado ao grupo de lavanda. }\end{array}$ \\
\hline 03 & $\begin{array}{l}\text { Effects of Aromatherapy } \\
\text { Combined with Music Therapy on } \\
\text { Anxiety, Stress, and Fundamental } \\
\text { Nursing Skills in Nursing } \\
\text { Students: A } \quad \text { Randomized } \\
\text { Controlled Trial }\end{array}$ & $\begin{array}{l}\text { Ensaio clínico } \\
\text { randomizado }\end{array}$ & Nível II & $\begin{array}{l}\text { Ouve redução significativa dos níveis de ansiedade } \\
\text { quando utilizada a aromaterapia combinada com } \\
\text { musicoterapia e melhora no desempenho de habilidades } \\
\text { fundamentais de enfermagem. }\end{array}$ \\
\hline 04 & $\begin{array}{l}\text { Massagem com aromaterapia: } \\
\text { efetividade sobre a ansiedade de } \\
\text { usuários com transtornos de } \\
\text { personalidade em internação } \\
\text { psiquiátrica }\end{array}$ & $\begin{array}{l}\text { Ensaio clínico } \\
\text { não controlado }\end{array}$ & Nível III & $\begin{array}{l}\text { Houve diminuição significativa da ansiedade dos } \\
\text { participantes quando utilizada a técnica através de } \\
\text { massagem. Considerando o uso da aromaterapia por } \\
\text { massagem. Ocorrendo redução dos parâmetros de } \\
\text { frequência respiratória e cardíaca. }\end{array}$ \\
\hline 05 & $\begin{array}{l}\text { Aromaterapia com ylang ylang } \\
\text { para ansiedade e autoestima: } \\
\text { estudo piloto }\end{array}$ & $\begin{array}{l}\text { Estudo piloto, de } \\
\text { campo, } \\
\text { experimental, } \\
\text { controlado }\end{array}$ & Nível II & $\begin{array}{l}\text { Considerando a avaliação intragrupo, antes e após a } \\
\text { intervenção, houve diferença significante para os três } \\
\text { grupos em especial na variável autoestima. }\end{array}$ \\
\hline 06 & $\begin{array}{l}\text { Benefícios das práticas } \\
\text { integrativas e complementares no } \\
\text { cuidado de enfermagem }\end{array}$ & $\begin{array}{l}\text { Revisão } \\
\text { Sistemática }\end{array}$ & Nível I & $\begin{array}{l}\text { Observa-se que o uso de práticas integrativas, como a } \\
\text { aromaterapia proporciona maior relaxamento, alívio da } \\
\text { ansiedade e diminuições dos sintomas de tal patologia, } \\
\text { além de diminuir a medicalização dos pacientes. }\end{array}$ \\
\hline 07 & $\begin{array}{l}\text { Eficácia da aromaterapia na } \\
\text { redução de níveis de estresse e } \\
\text { ansiedade em alunos de graduação } \\
\text { da área da saúde: estudo } \\
\text { preliminar }\end{array}$ & $\begin{array}{l}\text { Ensaio clínico } \\
\text { randomizado }\end{array}$ & Nível II & $\begin{array}{l}\text { Houve redução dos níveis de ansiedade em } 13 \% \text { e } 19 \% \text {, } \\
\text { e em particular de diminuição de } 24 \% \text {. }\end{array}$ \\
\hline
\end{tabular}




\section{Discussão}

A aromaterapia é a ciência que estuda a aplicação OE de maneira terapêutica, de forma que se utiliza as propriedades desses óleos para, principalmente, buscar estabelecer o equilíbrio do organismo, promovendo o bem estar e promoção de saúde tanto física quanto mental. A mesma tem se apresentado nos ramos da medicina como uma prática que pode trazer diversas vantagens para os pacientes, além de ser barata, podendo ser exercida por vários profissionais que compõe a equipe multidisciplinar (Gnatta et al. 2014; Domingos \& Braga, 2015).

Os OE são substâncias voláteis com mecanismos farmacológicos comprovados. Os mesmos são extraídos de plantas que possuem propriedades terapêuticas significativas e são utilizados principalmente na aromaterapia, sendo que mesmo que os $\mathrm{OE}$ e a aromaterapia não tenham os mecanismos de ação à nível fisiológico bem delimitados, achados comprovam que quando as substâncias presentes nesses quando absorvidas, essas moléculas sejam capazes de estimular a liberação de neurotransmissores, como ocorre na liberação da endorfina, produzindo sensações e bem estar e relaxamento (Gnatta et al. 2014; Gnatta et al. 2016).

Um estudo específico demonstrou que a aromaterapia é uma técnica capaz de proporcionar a redução de alguns sintomas característicos em pessoas com ansiedade, como elevação da frequência cardíaca (FC) e frequência respiratória (FR). De maneira que em tal pesquisa realizaram-se seis sessões e desde a primeira já foi evidenciado a redução desses parâmetros (Domingos \& Braga, 2015).

Autores pesquisaram a associação de aromas específicos que podem atuar na redução de sintomas ansiosos. Como é o caso de uma pesquisa que teve como objetivo verificar se o uso do OE de ylang ylang, por meio de aplicação cutânea ou inalatória, alterava a percepção da ansiedade de pacientes, da autoestima e dos parâmetros fisiológicos como pressão arterial (PA) e temperatura. Evidenciou-se que o uso de carbogel mais o OE de ylang ylang reduziu os escores de ansiedade e foi observado que tal redução perdurou-se até mesmo após o uso de tal aroma. Um grupo de pacientes (grupo 1) recebeu o gel com OE de ylang ylang a 2\%; o grupo 2, placebo, recebeu gel com essência de ylang ylang a $2 \%$ e o grupo 3 recebeu um aromatizador pessoal com OE de ylang ylang (Gnatta et al. 2014).

No início do estudo, a maioria dos participantes apresentava elevação na pressão arterial média (PAM) (acima de $95 \mathrm{mmHg}$ ) e após três meses de uso, os três grupos manifestaram redução em tal parâmetro. Nas seções de aplicação de ylang ylang, por massagem, com concentrações da substância acima de 20\%, conseguiu-se reduzir os níveis da PAM em 20 minutos de sessão. Além disso, o estudo apontou uma melhora da autoestima e no bem estar físico, mental e emocional dos pacientes (Gnatta et al. 2014).

Esses resultados acima vão de encontro com outro estudo que fez a aplicação por via inalatória de OE de alecrim e limão com o objetivo de reduzir os níveis de ansiedade de estudantes universitários. O estudo mostrou que em média 6 dos 18 participantes relataram melhora da concentração após a participação das sessões, isso pelo fato desses se sentirem mais calmos. Além de observar que a grande maioria dos participantes da pesquisa fizeram comentários positivos acerca da técnica e cerca de $85 \%$ desses utilizariam a aromaterapia novamente no futuro como terapia calmante (Wells et al. 2020).

Outros dois estudos que também analisaram a redução do nível da ansiedade com a utilização da aromaterapia evidenciaram que tal técnica se mostra eficaz, sendo que em um desses estudos apresentou importante significância estatística, na qual o nível de estresse, dos participantes, alcançou uma redução de até $24 \%$ e o da ansiedade, especificamente, alcançou um percentual de redução de até $19 \%$, destacando que nesse estudo foram utilizados em conjunto o OE de lavanda, laranja azeda, ylang ylang e cedro (Gnatta et al. 2011; Lyra et al. 2010).

Uma outra pesquisa analisou a redução da ansiedade de professores de enfermagem, com aplicação de variados tipos de aromas. Observou-se a redução do estresse de um percentual de 8,72\% para o grupo que estava fazendo uso do OE de 
lavanda e redução de $8,90 \%$ do que fazia uso do $\mathrm{OE}$ de ylang ylang. Além disso, a pesquisa apontou a diminuição de parâmetros como PA e FC, visto que esse último apresentou diminuição em todas as sessões de aromaterapia. Porém, é destacado em tal estudo que por mais que se observe tal redução, a mesma não atinge níveis de significância estatística (Dias et al. 2019).

Além de isolada, a aromaterapia também é estudada quanto ao seu efeito associada com outras práticas complementares, como é o caso de um estudo experimental randomizado que foi realizado com 98 estudantes de enfermagem da Faculdade Sungshin Women's University, localizada na Coréia do Sul. O estudo apontou a diminuição dos níveis de ansiedade de um percentual de mais de 50\%, quando utilizada a aromaterapia combinada com a musicoterapia, além de ser observado melhor desempenho em algumas atividades acadêmicas e em algumas habilidades fundamentais de enfermagem, após a terapia (Son et al. 2019).

Em relação a aplicação da aromaterapia por enfermeiros observa-se que tal prática pode ser utilizada por esses profissionais em sua atuação, visto que a mesma demonstra resultados significativos, é de baixo custo e não oferece riscos aos pacientes que recebem tal terapêutica. Essa terapia pode aumentar o vínculo e confiança entre profissional e paciente, o que melhora os resultados durante o tratamento em diversas condições (Mendes et al. 2019).

A aromaterapia é reconhecida como uma prática eficaz no controle da ansiedade, logo pode ser utilizada pelos enfermeiros como prática complementar ou alternativa no tratamento de desordens emocionais e psíquicas. Isso ocorre devido ao fato da aromaterapia, sendo em conjunto com outras práticas complementares ou não, demonstrar resultados calmantes e os pacientes tendem a se sentirem mais equilibrados e tranquilizados, podendo reduzir a quantidade de atividades intervencionistas e a medicalização (Mendes et al. 2019).

É importante destacar que tal técnica, quando utilizada de maneira adequada, pode alcançar resultados significativos. Mesmo assim, a partir da análise dos artigos selecionados, observou-se que a utilização de métodos como a aromaterapia para a redução de sintomas ansiosos em pacientes que possuem esse e outros tipos de transtornos mentais ainda não é uma temática tão estudada e as pesquisas são escassas.

\section{Conclusão}

Nesse estudo, observou-se grande importância da utilização da aromaterapia no tratamento para ansiedade, um método não farmacológico que reduz os níveis de ansiedade em portadores com tal distúrbio. Por serem substâncias voláteis e que atuam através de mecanismos de ação e por meio de várias vias, os óleos essenciais atuam na redução de níveis de estresse, tensão muscular, frequência cardíaca, frequência respiratória e pressão arterial, podendo assim agir como um mecanismo que aumenta o nível de relaxamento.

A aromaterapia não necessita de técnicas complexas, podendo ser aplicado por diversos profissionais, como exemplo o enfermeiro, e ser utilizado diariamente pelos pacientes como um tratamento coadjuvante contínuo.

Além do mais, não foram encontrados artigos que estudaram e relataram os possíveis efeitos colaterais da utilização dos óleos essenciais. Além disso, não foram encontradas pesquisas que descrevesse especificamente o mecanismo de ação de cada substância.

Foi observado, através de nosso estudo de revisão, escassez de pesquisas que apontam a utilização da aromaterapia, de maneira isolada, na redução dos níveis de ansiedade de pacientes com o diagnóstico confirmado. Além disso, só foi encontrado um artigo que fala da atuação do enfermeiro na utilização desse tipo de terapêutica. Isso demonstra que há necessidade de incentivos e de pesquisas nacionais voltadas à atuação de tal profissional na aromaterapia associada ao tratamento de pacientes com transtornos ansiosos. 
Dessa forma maneira, espera-se que tal pesquisa contribua no aperfeiçoamento de profissionais atuantes na área, fazendo com que diminua o número de tratamentos farmacológicos. Além disso, a presente pesquisa pode servir de subsídio para o desenvolvimento de outros estudos com metodologias diferentes, de forma que aumente o desenvolvimento científico na área.

\section{Referências}

APA. (2014). Manual diagnóstico e estatístico de transtornos mentais: DSM-5. American Psychiatric Association ed.5, Porto Alegre: Artmed.

Bernardo W. M. (2011). Importância da análise dos níveis de evidência publicados. Rev Assoc Med Bras. 57(1):1.

Brasil. (2018). Ministério da Saúde. Gabinete do Ministro. Portaria n 702, de 21 de março de 2018. Altera a Portaria de Consolidação n $2 /$ GM/MS, de 28 de setembro de 2017, para incluir novas práticas na Política Nacional de Práticas Integrativas e Complementares - PNPIC. Diário Oficial da União, Brasília, DF, 65 .

Dias S. S. Domingos T. S. \& Braga E. M. (2019). Aromaterapia para a ansiedade e estresse de professores de enfermagem. Rev enferm UFPE on line. 13:e240179.

Domingos T. S. \& Braga E M. (2015) Massagem com aromaterapia: efetividade sobre a ansiedade de usuários com transtornos de personalidade em internação psiquiátrica. Rev Esc Enferm USP. 49(3):453-9.

Galvão C. M. (2006). Níveis de Evidência. Acta Paul Enferm, 19(2):V.

Galvão T .F. Pansani T. S. A. \& Harrad D. (2015). Principais itens para relatar Revisões sistemáticas e Meta-análises: A recomendação PRISMA. Epidemiol. Serv. Saúde, 24(2).

Gnatta J. R. Piason P. P. Lopes C. L. B. C. Rogenski N.M.B. \& Silva M.J.P. (2014). Aromaterapia com ylang ylang para ansiedade e autoestima: estudo piloto. Rev Esc Enferm USP, 48(3):492-9.

Gnatta, J. R. Dornellas, E. V. \& Silva, M .J. P. (2011). O uso da aromaterapia no alívio da ansiedade. Acta Paul Enferm, 24(2):257-63.

Gnatta, J. R. Kurebayashi, L. F. S. Turrini, R. N. T. \& Silva, M. J. P. (2016). Aromaterapia e enfermagem: concepção histórico-teórica. Rev. esc. enferm. USP, 50( 1 ): 127-133.

Hoare, J. (2010). Guia completo de aromaterapia. São Paulo: Editora Pensamento, ed. 1.

Leonardo, B. C. Cunha, D. F. Sakae, T. M. \& Kemor, K.V. T. (2017). Prevalência de transtornos mentais e utilização de psicofármacos em pacientes atendidos em um ambulatório médico de especialidades. Revista da Associação de Medicina Catarinense de Medicina, 46(2): 39-52.

Lyra, C. S. Nakai, L. S. \& Marques, A. P . (2010). Eficácia da aromaterapia na redução de níveis de estresse e ansiedade em alunos de graduação da área da saúde: estudo preliminar. Fisioterapia e Pesquisa, 17(1):13-7.

Machado, B. F. M. T. \& Júnior, A. F. (2011). Óleos essenciais: Aspectos gerais e usos em terapias naturais. Cadernos Acadêmicos, 3(2):105-127.

Malta, A. S. \& Lemos, L. M. A. (2019). O Uso de Óleos Essenciais no Tratamento do Estresse. Revista Multidisciplinar e de Psicologia, 13(48):54-5.

Mendes, D. S. Moraes, F. S. Lima, G. O. Silva, P. R. \& Cunha, T.A . Crossetti, M. G. O, et al. (2019). Benefícios das práticas integrativas e complementares no cuidado de enfermagem. Journal Health NPEPS, 4(1):302-318.

Mendes, K. D .S. Silveira, R. C. C .P. \& Galvão, C. M. (2013). Revisão integrativa: método de pesquisa para a incorporação de evidências na saúde e na enfermagem. Revista Texto e Contexto de Enfermagem, 17(4):758-764.

Onocko-Campos, R. T. (2019). Revisão integrativa: Saúde mental no Brasil: avanços, retrocessos e desafio. Revista Caderno de Saúde Pública, 35(11).

Organização Pan-Americana da Saúde (OPAS). (2017). Aumenta o número de pessoas com depressão no mundo. Brasília, DF, Brasil.

Santos, C. M. C. Pimenta, C. A. M. \& Nobre, M. R. C. (2007). A estratégia PICO para construção da pergunta de pesquisa e busca de evidências. Revista Latino-americana de Enfermagem, 15(3).

Pereira, F. L .R. Medeiros, S. P. Salgado, R. G. F. Castro, J. N. A. \& Oliveira, A. M. N. (2019). Manifestações de ansiedade vivenciadas por estudantes de enfermagem. Rev Fun Care Online, 11(4):880-6.

Son, H.K. So, W. \& Kim, M. (2019). Effects of Aromatherapy Combined with Music Therapy on Anxiety, Stress, and Fundamental Nursing Skills in Nursing Students: A Randomized Controlled Trial. Int. J. Environ. Res. Public Health, 16:1-10.

Souza, M. T. Silva, M. D. \& Carvalho, R. (2010). Revisão integrativa: o que é e como fazer. Revista Einstein, 8(1):102-6.

Wells, B. M. Nightingale, L. M. Derby, D. C. Salsbury, S. A. \& Lawrence, D. (2020). Aromatherapy for test anxiety in chiropractic students: A feasibility study. J Chiropr Educ., 00(0). 